\title{
High risk of septic complications following surgery for Crohn's disease in patients with preoperative anaemia, hypoalbuminemia and high CRP
}

\author{
Ahmed S. Ghoneima ${ }^{1}$ (D) Karen Flashman $^{1} \cdot$ Victoria Dawe $^{1} \cdot$ Eleanor Baldwin $^{1} \cdot$ Valerio Celentano $^{1,2}$
}

Accepted: 11 September 2019/Published online: 8 November 2019

(C) The Author(s) 2019

\begin{abstract}
Aim Bowel resection in Crohn's disease still has a high rate of complications due to risk factors including immune suppression, malnutrition and active inflammation or infection at the time of operating. In this study, we use serological levels and inflammatory markers to predict the potential of complications in patients undergoing resections for complicated Crohn's disease.

Methods All patients undergoing laparoscopic bowel resection for Crohn's disease from 5th of November 2012 to 11 th of October 2017 were included in this retrospective observational study. Patients were divided into 4 groups scoring $0,1,2$ or 3 depending on their pre-operative haemoglobin concentration $(\mathrm{Hb}), \mathrm{C}$-reactive protein $(\mathrm{CRP})$ and albumin (Alb) where 1 point was given for an abnormal value in each as detailed in the definitions. They were then grouped into a low risk group comprised of those scoring 0 and 1, and a high risk group for those scoring 2 and 3 and data was collected to compare outcomes and the incidence of septic complications.

Results Seventy-nine patients were included. Eleven (13.9\%) and 2 (2.5\%) patients had 2 or 3 abnormal values of CRP, Alb and $\mathrm{Hb}$ and were categorized as high risk. High risk patients had a significantly higher rate of post-operative septic complications $(30.7 \%)$ compared with low risk patients $(10.6 \%) p$ value $<0.0001$.

Conclusion Pre-operative CRP, haemoglobin and albumin can serve as predictors of septic complications after surgery for Crohn's disease and can therefore be used to guide pre-operative optimisation and clinical decision-making.
\end{abstract}

Keywords Crohn's disease $\cdot$ Laparoscopic surgery $\cdot$ Colorectal surgery $\cdot$ Ileocaecal resection $\cdot$ Inflammatory bowel disease

\section{Introduction}

Crohn's disease (CD) is a chronic inflammatory bowel disease (IBD) that may involve any part of the alimentary tract from mouth to anus; it affects all layers of the intestine from mucosa to serosa and is often discontinuous along the longitudinal axis with a propensity for the distal small intestine and proximal large bowel. Although advances in the medical treatment of the disease have reduced the need for surgery in IBD over the past

Preliminary data from the same database was presented as a poster in the ACPGBI 2018 Annual Meeting, 09-11 July 2018, Birmingham, UK

Ahmed S. Ghoneima

ahmed.seif.ghoneima@doctors.org.uk

1 Colorectal Surgery Unit, Queen Alexandra Hospital-Portsmouth Hospitals NHS Trust, Portsmouth, UK

2 University of Portsmouth, Portsmouth, UK
60 years [1], $50 \%$ of $\mathrm{CD}$ patients will require surgery after 10 years of diagnosis [1]. Indications for surgery in $\mathrm{CD}$ include [2] bowel perforations, intra-abdominal abscess formation, gastrointestinal bleeding, symptomatic fibrotic stricture, enteric fistulae, small bowel or colorectal cancer on top of long-standing inflammatory disease, persistent inflammation with symptoms refractory to medical therapy and disease limited to the terminal ileum after considering risks and benefit. Although bowel resection for $\mathrm{CD}$ is increasingly being undertaken laparoscopically with better recovery and shorter hospital stays compared with open operations [3], surgery in CD patients still has a high rate of complications [4-6] due to risk factors including immune suppression [7, 8], malnutrition [8,9] and active inflammation or infection at the time of operating $[8,9]$. Knowledge of risk factors and the ability to foresee complications pre-operatively is therefore necessary in $\mathrm{CD}$ patients undergoing surgical treatment. In this study, we use pre-operative serological levels and inflammatory markers to predict the potential of complications in patients undergoing resections for complicated $\mathrm{CD}$. 


\section{Methods}

\section{Study design}

All patients undergoing ileocaecal, small bowel and colonic resections on elective theatre lists for $\mathrm{CD}$ from 5 th of November 2012 to 11th of October 2017 were included in this retrospective observational study based on a prospectively maintained database. The study was designed according to the STROBE checklist. All patients undergoing open, robotic or hand-assisted surgery for $\mathrm{CD}$ were excluded as were patients undergoing emergency operations and operations that did not entail a resection (e.g., stricturoplasties only).

The indication for surgery was discussed at a dedicated IBD multi-disciplinary team meeting (MDT) and pre-operative assessment included colonoscopy, MRI enterography and intestinal ultrasound. Before the planned surgical procedure, the patients were reviewed in a dedicated preassessment outpatient clinic where patients with significant weight loss prior to surgery were referred to the dietitian team for optimisation and iron infusion was consider if the value of the haemoglobin concentration $(\mathrm{Hb})$ was less than $<10 \mathrm{~g} / \mathrm{dL}$.

Patients were divided to 4 groups scoring $0,1,2$ and 3 depending on their pre-operative $\mathrm{Hb}, \mathrm{C}$-reactive protein (CRP) and albumin (Alb) where 1 point was given for an abnormal value in each as detailed in the definitions. They were then grouped into a low risk group comprised of those scoring 0 and 1, and a high risk group for those scoring 2 and 3 and data was collected to compare outcomes and the incidence of complications.

\section{Definitions}

Pre-operative blood tests are defined as values obtained during pre-operative assessment within 1 week from the operation. Abnormal values were defined as $\mathrm{Hb}<12 \mathrm{~g} /$ $\mathrm{dL}$ [5], CRP $>30 \mathrm{mg} / \mathrm{L}$ and albumin $<35 \mathrm{~g} / \mathrm{L}$ [4, 5]. Post-operative complications are defined as occurring within 30 days of surgery. Intra-abdominal septic complications (IASC) is a term that collectively describes intra-abdominal abscesses, pus collections and anastomotic leaks [9].

\section{Primary and secondary outcomes}

The primary outcome was the rate of septic complications including wound infections (SSI), anastomotic leaks and intra-abdominal collections.

Secondary outcomes included post-operative length of stay, readmission and reoperation rate.

\section{Statistical analysis}

Categorical variables are presented as frequency or percentage and were compared with the use of the chi-square test or Fisher's exact test, as appropriate. Continuous variables are presented as mean ( \pm standard deviation) or median (range) and were compared with the use of Student's $t$ test. The Mann-Whitney $U$ test was used for continuous, not normally distributed outcomes.

\section{Ethics}

The study is conducted in accordance with the principles of the Declaration of Helsinki and 'good clinical practice' guidelines. Informed consent has been obtained from the patients.

\section{Results}

Eighty-eight patients underwent elective laparoscopic bowel resection for $\mathrm{CD}$ during the study period, and after the exclusion of 9 patients in whom the pre-operative CRP and/or albumin were not available, 79 patients ( 24 male, 55 female) were included in the study. Median age was 38 (28-49) and 30 patients $(38 \%)$ had history of previous abdominal surgery performed via laparotomy for non-CD-related reasons.

The indications for surgery were fibrostenotic disease in 50 patients $(63.3 \%)$ and penetrating disease with entero-enteric, entero-colic or entero-urinary fistulae in 29 patients $(36.7 \%)$. Six procedures $(7.6 \%)$ were converted to open and 13 patients (16.4\%) had an ileostomy fashioned at the time of the surgery. Median LOS was 9 days (7.5-9.5) and 4 patients were readmitted (5\%) while 3 patients required reoperation (3.8\%). There were 24 post-operative complications (30\%).

Almost $50 \%$ of our patient population had one or more pre-operative abnormal values of $\mathrm{Hb}$, albumin and CRP. Patients with none or one pre-operative abnormal blood results (low $\mathrm{Hb}$, low Alb and high CRP) were categorized as low risk, while patients with two or three abnormal results of were defined as high risk, and post-operative outcomes in these two populations are presented in Table 1, demonstrating a statistically significant higher rate of intra-abdominal septic complications in patients with two or more pre-operative blood results abnormalities.

\section{Discussion}

Surgery for CD carries high risks of complications including [4] wound infections, anastomotic leak and intraabdominal sepsis $[8,9]$. Ileocaecal resections for $\mathrm{CD}$ have a similar complication profile to those undergoing a similar operation for 
Table $1 \quad 30$-day postoperative complications. Data presented as number (\%)

\begin{tabular}{llll}
\hline Group (score) & Low risk (score 0-1) & High risk (score 2-3) & $p$ value \\
\hline Total number of patients & 66 & 13 & \\
& No risk factors: $40(50.63 \%)$ & 2 risk factors: $11(13.92 \%)$ & 3 risk factors: 2(2.53\%) \\
Overall complications & 1 risk factor: $26(32.91 \%)$ & $7(53.85 \%)$ & $<(46.15 \%)$ \\
Surgical site infection & $17(25.76 \%)$ & $4(30.77 \%)$ & $<0.0001$ \\
Intra-abdominal septic complications (IASC) & $7(10.6 \%)$ & $1(7.69 \%)$ & $<0.0001$ \\
Bleeding & $7(10.6 \%)$ & $7(53.8 \%)$ & $\mathrm{ns}$ \\
Ileostomy formation required & $3(4.55 \%)$ & 11 & $<0.0001$ \\
Mean length of hospital stay in (Days) & $6(9 \%)$ & $5(38.4 \%)$ & $\mathrm{n}$ \\
Pre-operative anti-TNF treatment & 8.6 & $4(30.7 \%)$ & $\mathrm{ns}$ \\
Pre-operative steroids treatment & $24(36.4 \%)$ & $14(21.2 \%)$ & $\mathrm{ns}$ \\
\hline
\end{tabular}

cancer [6], despite patients being around 30 years younger with far less co-morbidity.

The tendency for formation of fistulae, abscesses, and large phlegmons, multifocal disease and thickened mesentery make CD resection technically challenging [10]; therefore, it should be undertaken in centres with high procedural caseloads and dedicated surgeons as core members of the IBD multidisciplinary team [11] with the aim to prevent emergency surgery, postoperative complications and recurrence.

Risk factors leading to high complication rates in CD surgery include immune suppression $[4,10]$ as corticosteroid therapy within 3 months before surgery [7, 8] and higher pre-operative serum biological levels, malnutrition measured by loss of $>10 \%$ of weight in 6 months or albumin levels $<25 \mathrm{~g} / 1$ [8, 9], repeated episodes of disease flare up [10] and the presence of fistulas and/or abscesses at the time of operating [8,9]. The presence of more than one of these factors compounds the risk of complications, specifically intra-abdominal septic complications, where the presence of one or two risk factor leads to an incidence of $14-16 \%$, three risk factors leads to $26-$ $29 \%$ and four leads to $50-100 \%[8,9]$.

Our study found that a significant proportion of patients undergoing elective surgery for primary ileocaecal CD had pre-operative blood test abnormalities, as almost $50 \%$ of our patients had one or more abnormal preoperative albumin, $\mathrm{Hb}$ or CRP. The presence of anaemia and hypoalbuminemia, or high CRP pre-operatively has a cumulative effect on the risk of incidence of post-operative intra-abdominal septic complications and prolonged post-operative length of hospital stay. We found a high rate of post-operative septic complications following ileocolic CD surgery, with 11 patients out of 79 (13.9\%) experiencing intra-abdominal sepsis such as anastomotic leaks and intra-abdominal collections, similar to the previous studies [12].

Our results suggest that abnormal pre-operative serological markers can reflect risk factors and in turn predict post- operative complications. Knowledge of these risk factors may be beneficial when consenting patients for surgery regarding risks and expectations of the post-operative recovery period as well as guiding the need of pre-operative optimization e.g. entral/parenteral nutrition and decisions of intervention e.g. formation of defunctioning stoma rather than anastomosis.

Our study did not include patients having emergency surgery or surgery for recurrent CD as these two groups are known for an increased risk of overall complications and post-operative anastomotic leaks and collection. Our database did not include patients undergoing open surgery which is a limitation of this study. Nevertheless, our unit has been one of the major contributing centres for the national laparoscopic surgery training program in Great Britain (LAPCO) and all major laparoscopic resections are performed according to a standardized technique. Open surgery is now rarely offered in our minimally invasive unit in the elective setting and therefore the number of patients not recruited as having undergone elective open surgery for primary ileocaecal CD is likely to be irrelevant.

Another limitation of our study is that no direct patientreported outcome measures have been assessed and no data have been collected on rate and length of post-operative admission to intensive care, which could be set as objectives for further prospective studies with patients stratified according to disease phenotype, pre-operative medical treatment and CD activity scores.

\section{Conclusion}

Our study confirms that pre-operative CRP, haemoglobin and albumin can serve as predictors of septic complications after surgery for Crohn's disease and can therefore be used to guide pre-operative optimisation and clinical decision-making. 


\section{Compliance with ethical standards}

Conflict of interest The authors declare that they have no conflict of interest.

Open Access This article is distributed under the terms of the Creative Commons Attribution 4.0 International License (http:// creativecommons.org/licenses/by/4.0/), which permits unrestricted use, distribution, and reproduction in any medium, provided you give appropriate credit to the original author(s) and the source, provide a link to the Creative Commons license, and indicate if changes were made.

\section{References}

1. Frolkis AD, Dykeman J, Negrón ME, Debruyn J, Jette N, Fiest KM, Frolkis T, Barkema HW, Rioux KP, Panaccione R, Ghosh S, Wiebe S, Kaplan GG (2013) Risk of surgery for inflammatory bowel diseases has decreased over time: a systematic review and meta-analysis of population-based studies. Gastroenterology. 145(5):996-1006

2. Strong S, Steele SR, Boutrous M, Bordineau L, Chun J, Stewart DB, Vogel J, Rafferty JF, Practice C (2015) Guidelines Committee of the American Society of Colon and Rectal Surgeons Clinical Practice Guideline for the Surgical Management of Crohn's disease. Dis Colon Rectum 58(11):1021-1036

3. Duepree HJ, Senagore AJ, Delaney CP, Brady KM, Fazio VW (2002) Advantages of laparoscopic resection for ileocecal Crohn's disease. Dis Colon Rectum 45(5):605-610

4. Guo K, Guanwei J, Qiongyuan L, Xiuwen H, Zhiwei W, Gefei W, Guosheng W, Huajian G, Zhiwu R, Hong Jieshou L (2017) Risk factors of surgical site infections in patients with Crohn's disease complicated with gastrointestinal fistula. Int $\mathrm{J}$ Color Dis 32(5):635-643
5. Liu S, Miao J, Wang G, Wang M, Wu X, Guo K, Feng M, Guan W, Ren J (2017) Risk factors for postoperative surgical site infections in patients with Crohn's disease receiving definitive bowel resection. Sci Rep 7(1):9828-9828

6. Bone L, Pinkney T et al. (2014) A comparison of the post-operative course of Crohn's disease and cancer patients undergoing similar right-sided bowel operations. Int J Surg, Volume 12, Pages S32-S32

7. Fumery M, Seksik P, Chirica M, Gornet JM, Boschetti G, Cotte E, Buisson A, Dubois A, Nachury M, Zerbib P, Chafai N, Stefanescu C, Panis Y, Marteau PR, Pautrat K, Sabbagh C, Jerome F, Alle M (2015) Postoperative complications after ileo-caecal resection for Crohn's disease: a prospective multicentre study gastroenterology. 148(4) Suppl 1):S132-S133

8. Alves A, Panis Y, Bouhnik Y, Pocard M, Vicaut E, Valleur P (2007) Risk factors for intra-abdominal septic complications after a first ileocecal resection for Crohn's disease: a multivariate analysis in 161 consecutive patients. Dis Colon Rectum 50:331-336

9. Yamamoto T, Allan RN, Keighley MRB (2000) Risk factors for intra-abdominal sepsis after surgery in Crohn's disease. Dis Colon Rectum 43:1141-1145

10. Celentano V, Finch D, Forster L, Robinson JM, Griffith JP (2015) Safety of supervised trainee performed laparoscopic surgery for inflammatory bowel disease. Int J Color Dis 30:639-644

11. Morar PS, Hollingshead J, Bemelman W, Sevdalis N, Pinkney T, Wilson G, Dunlop M, Davies RJ, Guy R, Fearnhead N, Brown S, Warusavitarne J, Edwards C, Faiz O (2017) Establishing key performance indicators [KPIs] and their importance for the surgical management of inflammatory bowel disease results from a PanEuropean, Delphi Consensus Study. J Crohns Colitis 11(11): $1362-1368$

12. Rosenfeld G, Qian H, Bressler B (2013) The risks of post-operative complications following pre-operative infliximab therapy for Crohn's disease in patients undergoing abdominal surgery: a systematic review and meta-analysis. J Crohns Colitis 7(11):868-877

Publisher's note Springer Nature remains neutral with regard to jurisdictional claims in published maps and institutional affiliations. 